\title{
MENINGITE CRIPTOCÓCICA: ASPECTOS CLÍNICOS, EVOLUTIVOS E HISTOPATOLÓGICOS SEGUNDO A CONDIÇÃO PREDISPONENTE
}

\author{
Adhemar M. Fiorillo ${ }^{\dagger}$, Regina Helena P. Lima, \\ Roberto Martinez, Carlos E. Levy, \\ Osvaldo M. Takayanagui e José Barbieri Neto
}

\begin{abstract}
Dezessete casos consecutivos de meningite criptocócica foram analisados depois de divididos em 3 grupos: I. três pacientes sem imunodeficiência; II. seis pacientes com doenca primária: neoplasia (3), diabetes (2) e alcoolismo (1); III. oito pacientes que desenvolveram criptococose depois de 18 a 67 meses de submetidos a transplante renale imunossupressão medicamentosa. A duração mediana da sintomatologia antes do diagnóstico foi maior no Grupo II (53 dias) do que nos Grupos I (25 dias) ou III ( 28 dias). Rigidez de nuca, comprometimento de pares cranianos e papiledema foram mais comuns no Grupo I do que nos Grupos II ou III, mas febre e sinais neurológicos focais foram observados apenas nos doentes destes últimos grupos. Apesar do predomínio de linfócitos na maioria dos casos, foi mais freqüente nos pacientes com rim transplantado a presença de neutrófilos no líquor cefalorraquiano. A mortalidade tardia foi maior nos pacientes com doenca primária e, dos casos que receberam tratamento antifúngico efetivo, tiveram melhor prognóstico os pacientes com transplante renal. Encontrou-se o criptococo nos tecidos dos 8 casos necropsiados, notando-se também a formação de granulomas, exceto em 2 doentes do Grupo II. As diferenças observadas entre os Grupos sugerem que o quadro clínico, a evolução e os achados necroscópicos da meningite criptocócica são modificados de acordo com o tipo de imunodeficiência do paciente.
\end{abstract} nista.

Palavras-chaves: Criptococose. Meningite. Transplante renal. Intecção oportu-

A infecção produzida pelo Cryptococcus neoformans provoca amplo espectro de resposta do hospedeiro, compreendendo desde casos assintomáticos à meningoencefalite ou à criptococose genèralizada ${ }^{11}$. A virulência do agente apresenta importância menor na patogenia da doença quando comparada ao estado imune do hospedeiro, sendo que os quadros mais graves usualmente são os dos pacientes com imunidade celular comprometida ${ }^{14}$. Em virtude do uso mais generalizado de drogas imunossupressoras, a população de indivíduos com alterações iatrogênicas de defesa imune tem aumentado e, com ela, tem crescido a freqüencia das infecções criptocócicas, em especial a meningite ${ }^{9} 151819$. A casuistica brasileira compreende um número signifi-

Trabalho dos Departamentos de Clínica Médica e de Patologia do Hospital das Clínicas da Faculdade de Medicina de Ribeirão Preto/Universidade de São Paulo, Ribeirāo Preto, SP.

† Falecido em 13/07/89.

Correspondência para: Dr. Roberto Martinez. Departamento de Clínica Médica. Faculdade de Medicina.

14049 Ribeirão Preto, SP.

Recebido para publicaçào em 02/10/87. cativo de pacientes e, embora haja telatos de casos de criptococose pulmonar, a forma meningítica ou meningoencefalítica tem sido a mais freqüente 571720 .

No presente relato, correspondente a casos de meningite criptocócica, buscou-se avaliar a presença e o tipo de condição predisponente como fatores modificadores da infecção. Para isto, realizou-se uma análise comparativa de aspectos clínicos, evolutivos, histopatológicos e do líquido cefalorraquiano (LCR) em 3 grupos de pacientes: sem imunossupressão aparente ou com imunodeficiência relacionada a doença coexistente ou ao uso de imunossupressores após transplante renal.

\section{MATERIAL E MÉTODOS}

No periodo de 1969 a 1985 foram internados 17 doentes com meningite ou meningoencefalite criptocócica no Serviço de Moléstias Infecciosas do Hospital das Clinicas da Faculdade de Medicina de Ribeirão Preto (Estado de São Paulo). Efetuou-se a análise das manifestações e da evolução da criptococose nestes doentes após dividí-los em três grupos, de acordo com a existência e o tipo de condição predisponente à infecção fúngica: Grupo I - sem 
Fiorillo AM, Lima RHP, Martinez, R, Levy CE, Takayanagui OM, Barbieri Neto J. Meningite criptocócica: aspectos clínicos, evolutivos e histopatológicos segundo a condição predisponente. Revista da Sociedade Brasileira de Medicina Tropical 23: 19-25, jan-mar, 1990.

imunodeficiência aparente: 3 doentes do sexo feminino, com idade entre 27 e 40 anos. Grupo II - com doença primária potencialmente causadora de imunodeficiência secundária: 6 doentes com idade entre 17 e 69 anos, sendo 5 do sexo masculino e 1 do feminino. Grupo III - em uso de drogas imunossupressoras depois de submetidos a transplante renal: 8 doentes com idade entre 23 e 41 anos, 6 do sexo masculino e 2 do feminino. A imunossupressão vinha sendo feita com azatioprina ( 3 a $5 \mathrm{mg} / \mathrm{kg}$ de peso/dia, inicialmente, mantendo-se após 1 a $2 \mathrm{mg} / \mathrm{kg} / \mathrm{dia})$ e prednisolona ou prednisona $(1,2$ a $1,5 \mathrm{mg} / \mathrm{kg}$ de peso/dia no inicio, reduzindo-se gradativamente até a dose de manutenção de 0,25 a $0,50 \mathrm{mg} / \mathrm{kg} / \mathrm{dia}$ ).

$O$ diagnóstico da criptococose foi realizado pelo teste da tinta da China (tinta Nankin - Rotring Pelikan) e pela cultura do LCR (meios de cultura BHI e ou Sabouraud), identificando-se o $C$. neoformans pela assimilação de carbohidratos e pelo teste da urease. $\mathrm{O}$ método direto permitiu a demonstração dessa levedura no LCR em 13 casos e no escarro em 1 caso; isolou-se o criptococo na cultura de LCR de 14 pacientes e do sangue de 2 pacientes. $O$ exame necroscópico foi o único método diagnóstico do caso no 9 (Grupo II).

O tratamento especifico para 13 doentes consistiu de anfotericina B em dose total de $830 \mathrm{mg}$ a $4790 \mathrm{mg}, 9$ dos quais receberam também 5 - fluorci- tosina, em dose diária de $3 \mathrm{~g} \mathrm{a} 8 \mathrm{~g}$ e dose total de $136 \mathrm{~g} \mathrm{a}$ $616 \mathrm{~g}$. Três outros pacientes (n.s 10,13 e 14 do Grupo III) faleceram depois de poucos dias do início do tratamento. O doente n. 9 do Grupo II não recebeu terapia anti-fúngica.

As lâminas histopatológicas correspondentes aos casos autopsiados foram revisadas quanto à presença de leveduras e de processo inflamatório e formação de granulomas. Além da coloração hematoxilinaeosina, utilizou-se também das colorações mucicarmin e PAS para aumentar a sensibilidade da pesquisa de fungos em tecidos sem inflamação.

\section{RESULTADOS}

\section{Tipo e duração da condiçāo predisponente}

No grupo II, a condição predisponente consistiu de doença neoplásica ( 3 casos), diabetes melito ( 2 casos) e alcoolismo ( 1 caso), os quais estavam presentes desde 4 meses a muitos anos antes da criptococose (Tabela 1). A radioterapia efetuada no paciente no 4 e a quimioterapia no paciente no 9 podem ter contribuido para o surgimento da infecção oportunista. No Grupo III, os 8 doentes desenvolveram criptococose depois de 18 a 67 meses (mediana $=41$ meses) do transplante renal e de imunossupressão medicamentosa (Tabela 1).

Tabela 1 - Meningite criptocócica: dados clínicos e epidemiológicos de doentes sem imunossupressão (Grupo I), com doença primária associada (Grupo II) ou com imunossupressão após transplante renal (Grupo III).

\begin{tabular}{|c|c|c|c|c|c|c|}
\hline \multirow{2}{*}{ Grupo } & \multirow{2}{*}{ Paciente } & \multirow{2}{*}{$\begin{array}{l}\text { Idade } \\
\text { (anos) }\end{array}$} & \multirow{2}{*}{ Sexo } & \multicolumn{2}{|c|}{ Fator predisponente } & \multirow{2}{*}{$\begin{array}{l}\text { Tempo de doença } \\
\text { (dias) }\end{array}$} \\
\hline & & & & Tipo & Duração & \\
\hline I & 1 & 27 & $\mathrm{~F}$ & - & - & 25 \\
\hline \multirow[t]{2}{*}{$(n=3)$} & 2 & 40 & $\mathrm{~F}$ & - & - & 18 \\
\hline & 3 & 39 & $\mathrm{~F}$ & $-\quad \quad \quad:$ & - & 25 \\
\hline II & 4 & 69 & M & Câncer de bexiga & 4 meses & 75 \\
\hline \multirow[t]{5}{*}{$(\mathrm{n}=6)$} & 5 & 17 & $\mathbf{F}$ & Diabetes melito & 11 anos & 20 \\
\hline & 6 & 44 & M & Diabetes melito & 36 meses & 365 \\
\hline & 7 & 38 & $\mathbf{M}$ & Doença de Hodgkin IIIA & 7 anos & 30 \\
\hline & 8 & 56 & M & Alcoolismo crônico & 47 anos & 180 \\
\hline & 9 & 64 & $\mathbf{M}$ & Leucemia linfóide aguda & 8 meses & 20 \\
\hline III & 10 & 23 & $\mathbf{M}$ & Transplante renal & 53 meses & 8 \\
\hline \multirow[t]{7}{*}{$(\mathrm{n}=8)$} & 11 & 35 & $\mathbf{M}$ & Transplante renal & 27 meses & 50 \\
\hline & 12 & 25 & $\mathbf{M}$ & Transplante renal & 19 meses & 8 \\
\hline & 13 & 24 & $\mathbf{M}$ & Transplante renal & 42 meses & 10 \\
\hline & 14 & 41 & $\mathbf{M}$ & Transplante renal & 44 meses & 90 \\
\hline & 15 & 32 & $\mathrm{~F}$ & Transplante renal & 18 meses & 90 \\
\hline & 16 & 36 & M & Transplante renal & 40 meses & 8 \\
\hline & 17 & 29 & $\mathrm{~F}$ & Transplante renal & 67 meses & 45 \\
\hline
\end{tabular}


Fiorillo AM, Lima RHP, Martinez, R, Levy CE, Takayanagui OM, Barbieri Neto J. Meningite criptocócica: aspectos clinicos, evolutivos e histopatológicos segundo a condição predisponente. Revista da Sociedade Brasileira de Medicina Tropical 23: 19-25, jan-mar, 1990.

\section{Periodo de doença}

O periodo entre o inicio da sintomatologia da criptococose e seu diagnóstico variou de 18 a 25 dias (mediana: 25 dias) no Grupo I, 20 a 365 dias (mediana: 53 dias) no Grupo II e 8 a 90 dias (mediana: 28 dias) no Grupo III (Tabela 1).

\section{Sintomatologia}

Conforme mostra a Tabela $2,94 \%$ dos doentes queixaram-se de cefaléia e $65 \%$ de náuseas e vômitos. Menos freqüentes, porém de maior importância no diagnóstico foram as alterações de comportamento (desorientação, confusão mental, apatia, agitação) em $47 \%$ dos casos, as alteraçôes do estado de consciència (sonolência, torpor, coma) em $35 \%$, a rigidez de nuca em $41 \%$, as alterações de nervos cranianos (paralisia facial, estrabismo, desvio ocular) em $24 \%$ e o papiledema em $29 \%$ dos doentes. Também foram mencionados ou observados: fotofobia. diplopia, crise convulsiva, hipo ou hiperreflexia, paresia, parestesia, distúrbio da marcha, alteração do equilibrio, afasia e incontinência urinária. Nenhuma das pacientes do Grupo I teve febre mas apresentaram rigidez de nuca, papiledema e alteração de nervos cranianos em maior proporção do que nos outros grupos. No Grupo III, inversamente, $75 \%$ dos doentes tinham febre, mas somente a minoria dos casos mostrava rigidez de nuca, papiledema e alteraçāo de pares cranianos. O Grupo II, embora com menor ocorrência de febre entre os pacientes, mostrou-se comparável ao Grupo III. O doente n. 16 (Grupo III) tinha apenas sintomatologia respiratória e nenhuma manifestação neurológica, sendo a infecção do sistema nervoso comprovada em exame liquórico.

Tabela 2 - Sintomatologia dos 17 pacientes com meningite criptocócica

\begin{tabular}{|c|c|c|c|c|c|c|c|c|}
\hline \multirow{3}{*}{ Sintomatologia } & \multicolumn{8}{|c|}{ Grupos de Pacientes } \\
\hline & \multicolumn{2}{|c|}{$\begin{array}{c}\text { I } \\
\text { Sem imunos- } \\
\text { supressão }\end{array}$} & \multicolumn{2}{|c|}{$\begin{array}{c}\text { II } \\
\text { Doença } \\
\text { primária }\end{array}$} & \multicolumn{2}{|c|}{$\begin{array}{c}\text { III } \\
\text { Transplante } \\
\text { renal }\end{array}$} & \multicolumn{2}{|c|}{ Total } \\
\hline & no & $\%$ & no & $\%$ & no & $\%$ & no & $\%$ \\
\hline Cefaléia & 3 & 100 & 6 & 100 & 7 & 88 & 16 & 94 \\
\hline Náuseas e vômitos & 2 & 67 & 3 & 50 & 6 & 75 & 11 & 65 \\
\hline Febre & 0 & 0 & 2 & 33 & 6 & 75 & 8 & 47 \\
\hline Alteração do comportamento & 1 & 33 & 3 & 50 & 4 & 50 & 8 & 47 \\
\hline Rigidez de nuca & 2 & 67 & 2 & 33 & 3 & 38 & 7 & 41 \\
\hline Alteração da consciência & 1 & 33 & 3 & 50 & 2 & 25 & 6 & 35 \\
\hline Papiledema & 2 & 67 & 2 & 33 & 1 & 13 & 5 & 29 \\
\hline Alteração de pares cranianos & 2 & 67 & 0 & 0 & 2 & 25 & 4 & 24 \\
\hline Alterações visuais & 1 & 33 & 0 & 0 & 2 & 25 & 3 & 18 \\
\hline Crise convulsiva & 0 & 0 & 1 & 17 & 1 & 13 & 2 & 12 \\
\hline Outras alterações neurológicas & 0 & 0 & 3 & 50 & 3 & 38 & 6 & 35 \\
\hline
\end{tabular}

\section{Líquido cefalorraquiano}

O exame do LCR foi realizado em 16 casos (Tabela 3). Dois doentes tinham mais de 1000 células $/ \mathrm{mm}^{3}$, mas os outros 14 apresentaram celularidade entre 33 e 328 células $/ \mathrm{mm}^{3}$. Predominaram as células mononucleares, com exceção dos pacientes nos 2 (Grupo I), 10, 11 e 13 (Grupo III). Hiperproteinorraquia moderada foi encontrada em 15/16 casos e a glicorraquia era inferior a $50 \mathrm{mg} \%$ em $7 / 16$ casos. Comparando os 3 grupos de doentes, verificouse tendência a maior proporção de neutrófilos no LCR dos pacientes do Grupo III. 
Fiorillo AM, Lima RHP, Martinez, R, Levy CE, Takayanagui ON, Barbieri Neto J. Meningite criplocócica: aspectos clinicos, evolutivos e histopatológicos segundo a condiçâo predisponente. Revista da Sociedade Brasileira de Medicina Tropical 23: 19-25, jan-mar, 1990.

Tabcla 3 Citologia e bioquımica do lıquido cefalorraquiano inicial de 16 doentes com meningite criptococica.

\begin{tabular}{|c|c|c|c|c|c|c|c|c|}
\hline Grupo & Paciente & $\begin{array}{l}\text { Nivel de } \\
\text { puncào }\end{array}$ & $\begin{array}{l}\text { No de celulas } \\
\left(\text { por } \mathrm{mm}^{3}\right)\end{array}$ & $\begin{array}{c}\text { Neutrofi- } \\
\operatorname{los}(\%)\end{array}$ & $\begin{array}{l}\text { Linfoci- } \\
\text { tos }(\%)\end{array}$ & $\begin{array}{l}\text { Monoci- } \\
\text { tos }(\%)\end{array}$ & $\begin{array}{l}\text { Proternas } \\
(\mathrm{mg} / \mathrm{dl})\end{array}$ & $\begin{array}{l}\text { Glicose } \\
\text { (mg/dl) }\end{array}$ \\
\hline \multirow[t]{4}{*}{ I } & 1 & LO & 175 & 1 & 98 & 1 & 108 & 71 \\
\hline & 2 & SO & 1830 & 49 & 48 & 0 & 176 & 17 \\
\hline & 3 & LO & 149 & 0 & 100 & 0 & 20 & 58 \\
\hline & Mediana: & & 175 & 1 & 98 & 0 & 108 & 58 \\
\hline \multirow[t]{6}{*}{ II } & 4 & SO & 75 & 0 & 100 & 0 & 69 & 47 \\
\hline & 5 & LO & 33 & 0 & 100 & 0 & 63 & 18 \\
\hline & 6 & LO & 126 & 0 & 93 & 7 & 149 & 45 \\
\hline & 7 & $\mathrm{SO}$ & 1600 & 0 & 98 & 2 & 105 & 40 \\
\hline & 8 & SO & 48 & 1 & 96 & 3 & 63 & 89 \\
\hline & Mediana: & & 75 & 0 & 98 & 2 & 69 & 45 \\
\hline \multirow[t]{9}{*}{ III } & 10 & SO & 44 & 76 & 22 & 2 & 63 & 55 \\
\hline & 11 & LO & 328 & 64 & 32 & 4 & 56 & 78 \\
\hline & 12 & SO & 37 & 0 & 100 & 0 & 33 & 62 \\
\hline & 13 & LO & 273 & 67 & 33 & 0 & 82 & 62 \\
\hline & 14 & SO & 121 & 15 & 85 & 0 & 97 & 67 \\
\hline & 15 & $\mathrm{SO}$ & 150 & 9 & 71 & 20 & 30 & 38 \\
\hline & 16 & LO & 62 & 0 & 100 & 0 & 54 & 54 \\
\hline & 17 & LO & 74 & 19 & 81 & 0 & 83 & 44 \\
\hline & Mediana: & & 98 & 17 & 76 & 0 & 60 & 59 \\
\hline
\end{tabular}

Obs.: Paciente no 2: LCR com 3\% de eosinofilos; Paciente no 9: näo foi colhido LCR; LO = lombar; $S O=$ suboccipital.

\section{Complicacooes}

Alem do agravamento das manifestaçoes da meningite em alguns casos, durante a hospitalização foram freqüentes as infecçöes respiratórias, de vias urinárias e outras infecções piogênicas, ocorrendo também hemorragia digestiva ( 3 casos) e insuficiência renal ( 1 caso). Outras complicaçōes decorreram mais diretamente da criptococose: hidrocefalia ( 5 casos), trombose da artéria cerebral anterior direita (1 caso), amaurose ( 1 caso) e paraplegia ( 1 caso) (Tabela 4). Não foram observadas diferenças quanto à incidência de complicações nos Grupo I, II e III.

Tabela 4 - Meningite criptocócica: complicaçōes, evolução e achados necroscópicos em doentes sem imunossupressão (Grupo I), com doença primaria associada (Grupo II) ou com imunossupressao após transplante renal (Grupo III).

\begin{tabular}{|c|c|c|c|c|}
\hline Grupo & Paciente & Complicaçōes & Evolução & Órgãos envolvidos necrópsia) \\
\hline \multirow[t]{3}{*}{ I } & 1 & Hidrocefalia & Cura & - \\
\hline & 2 & Broncopneumonia & Obito & Meninge, plexo, coroide, pulmão \\
\hline & 3 & $\begin{array}{l}\text { Paraplegia Infecção urinäria } \\
\text { Pneumonia - Insuficiência renal }\end{array}$ & Óbito & $\begin{array}{l}\text { Meninge, cérebro, medula espinhal, pul- } \\
\text { mão, vesicula, hipofise }\end{array}$ \\
\hline \multirow[t]{6}{*}{ II } & 4 & - & Cura & - \\
\hline & 5 & Hidrocefalia - Otite - Inf. urinária & Cura & - \\
\hline & 6 & $\begin{array}{l}\text { Hidrocefalia - Pneumonia - Meningite } \\
\text { bacteriana - Hemorragia digestiva }\end{array}$ & Obito & Necrópsia nào realizada \\
\hline & 7 & Infeção urinaria & Obito & Meninge. cerebro \\
\hline & 8 & - & Cura & 5 \\
\hline & 9 & Pneumonia - Choque séptico & Obito & Meninge, cerebro, pulmào, rim, pâncreas \\
\hline \multirow[t]{8}{*}{ III } & 10 & - & Obito & Meninge, cérebro, pulmão, coração \\
\hline & 11 & Amaurose & Cura & 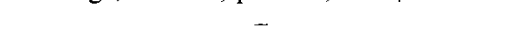 \\
\hline & 12 & $\begin{array}{l}\text { Sinusite - Inf. urinária - Hemorragia } \\
\text { digestiva }\end{array}$ & Cura & - \\
\hline & 13 & $\begin{array}{l}\text { Hidrocefalia - Trombose de artéria } \\
\text { cerebral anterior D }\end{array}$ & Obito & Meninge, encéfalo \\
\hline & 14 & - & Óbito & Necropsia não realizada \\
\hline & 15 & Hidrocefalia - Infecção urinária & Cura* & Meninge, cérebro, abscesso subcutâneo* \\
\hline & 16 & Pneumonia - Hemorragia digestiva & Óbito & Meninge, cerebro, pulmảo \\
\hline & 17 & Infecção urinaria & Cura & - \\
\hline
\end{tabular}

(*) Recidiva da criptococose e obito 19 meses apos o primeiro episodio. 
Fiorillo AM, Lima KHP, Martinez, R, Levy CE, Takayanagui OM, Barbieri Neto J. Meningite criptocócica: aspectos clínicos, evolutivos e histopatologicos segundo a condifão predisponente. Revista da Sociedade Brasileira de Medicina Tropical 23: 19-25, jan-mar, 1990.

\section{Mortalidade}

Considerando a evolução imediata de todos os doentes, verificou-se mortalidade de $67 \%$ no Grupo I, $50 \%$ no Grupo II e 50\% no Grupo III (Tabela 4). Tomando apenas os 13 doentes, efetivamente tratados, as taxas correspondentes de mortalidade alteramse para $67 \%(2 / 3), 40 \%(2 / 5)$ e $20 \%(1 / 5)$. No período entre 3 e 24 meses do final do tratamento faleceram mais 2 pacientes do Grupo II (no 4, de causa desconhecida, e n. 8 , após recidiva da criptococose) e 1 do Grupo III (no 15, de criptococose e septicemia)

\section{Achados necroscópicos}

Realizou-se a necrópsia em 8 pacientes (em um dos quais, após o segundo episódio de criptococose). $\mathrm{O}$ sistema nervoso estava comprometido nos 8 casos e em 6 deles o criptococo foi identificado também em outros tecidos, particularmente nos pulmòes ( 5 casos) (Tabela 4). Observou-se lesão granulomatosa ao menos em um dos tecidos infectados em $2 / 2$ casos do Grupo I e em 4/4 casos do Grupo III. Quanto ao grupo II, ou não havia reação inflamatória (paciente n. 7) ou esta era do tipo inespecifico (paciente no 9).

\section{DISCUSSÃO}

O conjunto de dados epidemiológicos, clínicos, laboratoriais e anátomo-patológicos da presente casuistica não difere do relatado anteriormente para outras séries de pacientes. Em $82 \%$ dos doentes a criptococose apresentou-se como infecção oportunista, quer no período de imunossupressăo após transplante renal, quer associando-se à moléstias preexistentes e potencialmente causadoras de defeitos na imunidade celular e na fagocitose. Dentre estas, são conhecidos fatores predisponentes da criptococose a doença de Hodgkin e outras doenças linfoproliferativas $^{9} 11$ e o diabetes melito ${ }^{2} 11$. O alcoolismo crônico e a cirrose hepática igualmente facilitam o desenvolvimento da criptococose 1621 . Estas doenças estavam presentes por tempo bastante variável antes do surgimento da infecção fúngica, desde alguns meses ate muitos anos antes. Nos 8 pacientes submetidos a transplante renal e imunossupressão com azatioprina e corticosteróides o periodo mediano entre a cirurgia e a instalação da meningite foi de 41 meses. Schroter e cols. 19 observaram periodo comparável (mediana igual a 48 meses) em 8 transplantados que tiveram esta infecção, mas nos 10 casos relatados por $G$ allis e cols. ${ }^{6}$ o período mediano foi somente de 11 meses. Em todos os 8 pacientes do Grupo III a criptococose manifestou-se no periodo pós transplante tardio, tal como delimitado por Rubin e cols. 18 .

Nos casos com doença coexistente foi mais longo o periodo mediano entre o início da sintomatologia e o diagnóstico da criptococose, cujas manifestações iniciais provavelmente foram confundidas com as da molesstia primária. $O$ curto periodo de sintomatologia antecedendo o diagnóstico ( 8 a 10 dias) verificado em 4 doentes com transplante renal pode ter resultado da maior atenção médica que recebem ou indicar formas graves de infecção, considerando-se que $3 / 4$ faleceram. A meningoencefalite criptocócica pode ter curso fulminante em pacientes imunossuprimidos apos transplante renal 6 , tipo de evolução que ocorreu em um desses 4 casos.

A sindrome de hipertensão intracraniana predominou no quadro clínico dos 3 grupos de doentes, como tem sido observado em outras séries de pacientes 4 21. Em relação ao grupo de pacientes não imunossuprimidos, os demais doentes apresentaram menor frequiência de rigidez de nuca, de papiledema $\mathrm{e}$ de envolvimento de pares cranianos, porem neles foram mais comuns a febre e vários tipos de distúrbios neurológicos. Estes dados sugerem alteração do processo reacional ao criptococo, incluindo reação imune celular de menor intensidade, menos fribrosante, nos pacientes com doença primária e nos que recebem drogas imunossupressoras. $O$ único paciente sem sintomatologia neurologica provavelmente tinha a meningite em fase de instalação, secundária ao foco pleuro-pulmonar e criptococemia.

A comparação da citologia do LCR mostrou tendência à maior participação dos neutrófilos na reação inflamatória dos doentes com transplante renal. Neutrófilos em proporções variadas também foram encontrados no LCR da maioria dos doentes de outras casuisticas 619 . Isto seria explicável pelo estágio inicial da meningite em alguns pacientes e, talvez, pela modificação da resposta inflamatória decorrente da ingestão crônica de croticosteróides. Entretanto, como se verificou com um caso do Grupo I, pacientes não imunossuprimidos, ocasionalmente apresentam predomínio de neutrófilos no LCR 1720 .

A mortalidade no conjunto dos pacientes $(52,9 \%)$ é semelhante às relatadas no Brasil por Nóbrega e cols. $13(50,0 \%)$, por Gonçalves e cols. ${ }^{7}$ $(55,6 \%)$ e por Kritski e cols. $10(50,0 \%)$. As complicaçōes, principalmente a hipertensão intracraniana e as infecções piogênicas constituem a causa imediata do óbito em pacientes com ou sem condição predisponente 12 14. Embora a evolução imediata tenha sido comparável nos 3 grupos de pacientes, nos meses seguintes aumentou o número de óbitos entre aqueles com doença primária pré-existente, confirmando o pior prognóstico da meningite criptocócica nesta situação clinica ${ }^{2} 3$. A mortalidade entre os pacientes com transplante renal $(50,0 \%)$ se aproxima da verificada em 17 casos do Hospital das Clínicas de Sào Paulo $(41,0 \%)^{12}$ e em outros países $(36,0-60,0 \%)^{68}$. 
Fiorillo AM, Lima RHP, Martinez, R, Levy CE, Takayanagui OM, Barbieri Neto J. Meningite criptocócica: aspectos clínicos, evolutivos e histopatológicos segundo a condição predisponente. Revista da Sociedade Brasileira de Medicina Tropical 23: 19-25, jan-mar, 1990.

Tomando-se apenas os 13 pacientes que receberam doses eficazes de antifúngicos, o prognóstico imediatamente após a terapia ou tardio foi mais favorável ao grupo com transplante renal.

A necrópsia de casos de meningoencefalite criptocócica tem mostrado que a infecção geralmente não se restringe ao sistema nervoso, encontrando-se o criptococo também nos pulmões e mesmo em outros tecidos onde sua presença não era suspeitada 711 . Nos doentes não imunossuprimidos e naqueles com transplante renal notou-se reação granulomatosa ao fungo, mas um paciente com doença de Hodgkin e outro com leucemia aparentemente ñao tinham condiçōes imunológicas para a formação do granuloma. $\mathrm{Na}$ criptococose pulmonar, Baker ${ }^{1}$ também notou a falta de reação granulomatosa nos doentes com deficiência imunológica.

Concluindo, ao serem cotejados os grupos de doentes sem imunossupressão, com doença coexistente ou submetidos a transplante de rim, foram observadas diferenças no quadro clínico e liquórico, na evolução e nos achados necroscópicos da meningoencefalite criptocócica as quais sugerem que certas caracteristicas da infecção fúngica possam ser alteradas pela presença e de acordo com o tipo de condição predisponente.

\section{SUMMARY}

Seventeen consecutive cases of cryptococcal meningitis diagnosed at Hospital das Clinicas of Ribeirào Preto Medical School (Sào Paulo StateBrazil) between 1969 and 1985 were reviewed. For analysis the patients were separated in 3 groups: $I$. three patients without immunodeficiency; II. six patients with associated disease: cancer (3), diabetes (2) and alcoholism (1); III. Eight renal transplant recipients that developed cryptococcosis after 18 to 67 months of immunosupression with steroids and azathioprine. The median interval between onset of symptoms and diagnosis of infection was greater in Group II (53 days) than in Groups I ( 25 days) or III (28 days). Neck stiffness, cranial nerve involvement and papilledema were more frequent in Group I than in Group II or III, but fever and focal neurological signs were observed only in patients of two last groups. Cerebrospinal fluid examination showed a mild lymphocytic pleocytosis in most patients, but transplant cases had polymorphonuclear cells more frequently. Late mortality was higher in patients with underlying disease and the prognosis was better for transplant patients that received effective antifungal therapy. Besides cryptococci, autopsy findings in 8 cases revealed granuloma formation in tissues, except in patients of 6 roup 11 ( 2 cases). The differences between the groups suggest that clinical characte- ristics, evolution and postmortem findings of the cryptococcal meningitis are changed according to type of immunodeficiency presented by the patient.

Key-words: Cryptococcosis. Meningitis. Renal transplant. Opportunistic infection.

\section{REFERÊNCIAS BIBLIOGRÁFICAS}

1. Baker RD. The primary pulmonary lymph node complex of cryptococcosis. The American Journal of Clinical Pathology 65:83-92, 1976.

2. Butler WT, Alling DW, Spickard A, Utz JP. Diagnostic and prognostic value of clinical and laboratory findings in cryptococcal meningitis. A follow-up study of forty patients. The New England Journal of Medicine 270:5967, 1964.

3. Diamond RD, Bennett JE. Prognostic factors in cryptococcal meningitis. A study in 111 cases. Annals of Internal Medicine 80:176-181, 1974.

4. Edwards VE, Sutherland JM, Tyrer JH. Cryptococcosis of the central nervous system: epidemiological, clinical and therapeutic features. Journal of Neurology, Neu rosurgery and Psychiatry 33:415-425, 1970.

5. Farias da Silva W, Travassos F, Codeceira Jr A, Rego JF. Criptococose do sistema nervoso central. Considerações a respeito de 5 casos. Neurobiologia 31:174-185, 1968.

6. Gallis HA, Bernan RA, Cate TR, Hamilton JD, Gunnels JC, Stickel DL. Fungal infection following renal transplantation. Archives of Internal Medicine 135:1163$1172,1975$.

7. Gonçalves AJR, Lopes PFA, Pinto AMM, Lazera M, Menezes JA, Cunha RQ, Pereira AA, Wanke B, Braga MP, Clemente HA, Duarte F. Criptococose: estudo de 27 casos observados no Hospital dos Servidores do Estado - INAMPS e no Hospital Estadual São Sebastiāo - Rio de Janeiro. Jornal Brasileiro de Medicina 46:43-63, 1984.

8. Hellman RN, Hinrichs J, Sicard G, Hoover R, Golden P, Hoffsten P. Cryptococcal pyelonephritis and disseminated cryptococcosis in a renal transplant recipient. Archives of Internal Medicine 141:128-130, 1981.

9. Hooper DC, Pruitt AA, Rubin RH. Central nervous system infection in the chronically immunosuppressed. Medicine 61:166-188, 1982.

10. Kritski AL, Gonçalves AR, Rozembaum R, Artus MC, Nogueira SA, Andrade EM, Clemente H. Criptococose do sistema nervoso central. Relato de seis casos e revisão da literatura. Revista Brasileira de Neurologia 22:171$178,1986$.

11. Lewis JL, Rabinovich $\mathrm{S}$. The wide spectrum of cryptococcal infections. American Journal of Medicine 53:315-322, 1972.

12. Nóbrega JPS. Contribuiçāo para o estudo do emprego da anfotericina B no tratamento da neurocriptococose em pacientes submetidos a transplante renal. Tese de Dou- 
Fiorillo $A M$, Lima RHP, Martinez, $R$, Levy CE, Takayanagui OM, Barbieri Neto J. Neningite criptocócica: aspectos clínicos, evolutivos e histopatológicos segundo a condição predisponente. Revista da Sociedade Brasileira de Medicina Tropical 23: 19-25, jan-mar, 1990.

toramento, Faculdade de Medicina da Universidade de Sāo Paulo, São Paulo, 1987.

13. Nóbrega JP, Livramento JA, Machado LR, SpinaFrança A. Criptococose do sistema nervoso central: avaliação da terapêutica por anfotericina B, 5-fluorocitosina e miconazole em 18 casos. Arquivos de NeuroPsiquiatria 37:28-33, 1979.

14. Perfect JR, Durack DT, Gallis HA. Cryptococcemia. Medicine 62:98-109, 1983.

15. Peterson PK, Ferguson R, Fryd DS, Balfour Jr HH, Rynasiewicz J, Simmons RL. Infectious diseases in hospitalized renal transplant recipients: a prospective study of a complex and envolving problem. Medicine 61:360-372, 1982.

16. Pincus MR, Silva-Hutner M, Rebatta G, Branwood AW. Disseminated cryptococcosis in an asymptomatic alcoholic man. Archives of Internal Medicine 141:796$797,1981$.
17. Reis-Filho JB, Neves AC, Zymberg ST, Oliveira RMC. $O$ liquido cefalorraquiano inicial nas meningencefalites por Cryptococcus neoformans. Revista do Instituto de Medicina Tropical de São Paulo 27:173-178, 1985.

18. Rubin RH, Wolfson JS, Cosimi AB, Tolkoff-Rubin NE. Infection in the renal transplant recipient. American Journal of Medicine 70:405-411, 1981.

19. Schröter GP, Temple DR, Husberg BS, Weil III R, Starzl TE. Cryptococcosis after renal transplantation: report of ten cases. Surgery 79:268-277, 1976.

20. Spina-França A, Silva JB. Diagnóstico e tratamento da criptococose do sistema nervoso central. Consideraçoes sobre 16 casos. Arquivos de Neuro-Psiquiatria (São Paulo) 26:115-126, 1968.

21. Yu YL, Nau YN, Woo E, Wong KL, Tse B. Cryptococcal infection of the nervous system. The Quarterly Journal of Medicine 66:87-96, 1988. 